\title{
Depth-averaged Simulation of Flows in Asymmetric Compound Channels with Smooth and Rough Narrow Floodplains
}

\section{Wisam Alawadi ${ }^{\mathrm{a}}$ and T.D. Prasad ${ }^{\mathrm{b}}$}

aSchool of Computing, Science \& Engineering; Newton Building, University of Salford, Salford, UK, M5 4WT; T: +44 (0)748 117 5640; email: w.a.a.k.alawadi@edu.salford.ac.uk.

${ }^{\mathrm{b}}$ School of Computing, Science \& Engineering; Newton Building, University of Salford, Salford, UK, M5 4WT; T: +44 (0)161 295 3644; email: d.p.tumula@ salford.ac.uk.

Corresponding author: Wisam Alawadi 


\title{
Depth-averaged Simulation of Flows in Asymmetric Compound Channels with Smooth and Rough Narrow Floodplains
}

\begin{abstract}
Depth-averaged hydrodynamic models are predominantly used in numerical simulations of compound channel flows. One of the most popular methods for the depth-averaged simulation is Shiono and Knight method (SKM). This method accounts for the effects of bed friction, lateral turbulence and secondary flows, via three key parameters $f, \lambda \& \Gamma$ respectively. The conventional expressions that are developed by Abril and Knight (2004) to calibrate these parameters are generally based on experiments in compound channels with wide floodplains. In this study, the application of SKM to an asymmetric compound channel with a narrow floodplain is examined in terms of the calibration requirements. Two sets of experiments that have smooth and rough floodplain and different flow depths are conducted and then simulated by SKM. In smooth floodplain cases, the results reveal that SKM model with the conventional calibration expressions of $f, \lambda$ and $\Gamma$ is reasonably capable of predicting the distributions of depth-averaged velocity and boundary shear stress in the main channel. However, in the floodplain region, the expressions recommended for calibrating $\Gamma$ need to be modified to improve the predicted results in that region. In cases of the rough floodplain, the results indicate that the lateral shearing is dominant over the secondary flow, so only the values $\lambda$ in the main channel need to be changed from its conventional values to improve the predictions.
\end{abstract}

Keywords: Asymmetric compound channel, SKM, Depth-averaged velocity, boundary shear stress, Narrow floodplains.

\section{Introduction}

Most rivers consist of a relatively deep channel with adjacent shallow floodplains so that the flow section takes a two-stage geometrical shape usually called a compound channel. The differences in water depth and bottom friction of the main channel and the floodplain regions cause the velocity in the floodplain to be lower than that in the main channel, generating a transverse gradient in streamwise velocity. This transverse gradient in the velocity and that in the bottom level of the channel create a mixing zone and cause exchange of mass and momentum. As consequence of that, the prediction of the key flow variables (such as the velocity and shear stress) in such a compound channel requires adequate modelling of flow features such as the 
momentum exchange and secondary flow cells that occurs at the main channel/floodplain interface (Shiono and Knight 1991; van Prooijen et al. 2005).

Many studies have concerned with the prediction of the depth-averaged velocity and boundary shear stress in compound channels, e.g. Ervine \& Ellis (1987); Shiono and Knight (1988, 1991); Wark et al. (1990); Lambert and Sellin (1996); Ervine et al. (2000); Spooner \& Shiono (2003); Van Prooijen et al. (2005) and Yang et al. (2012). Based on these studies, a number of twodimensional approaches for modelling the depth-averaged velocity and the boundary shear stress distributions have been developed. However, the methodology proposed by Shiono and Knight (1991), which is based on Reynolds averaged Navier-Stokes (RANS) equation, is the most popular method and is widely used for modelling different cases associated with the compound channel flows. Therefore, this paper concentrates only on the application of the SKM methodology to asymmetric compound channels that are considered here.

The Shiono and Knight method (SKM) provides an analytical solution for the lateral distributions of depth-averaged velocity and bed shear stress, accounting for the effects of the bed friction, lateral eddy viscosity, and secondary flow cells by means of three hydraulic parameters ( $f$ for friction, $\lambda$ for eddy viscosity, and $\Gamma$ for secondary flow). Based on the SKM method, many investigations have been undertaken into flow in straight and meandering compound channels. For example, the SKM method has been applied to straight prismatic channels by Shiono \& Knight (1991); Abril and Knight (2004); Liao and Knight (2007); Tang \& Knight (2008a); Knight \& Tang (2008); Seckin et al. (2009); and Devi \& Khatua (2016). Attempts have also been undertaken to use the SKM in modelling meandering channels (Liu et al. 2014) and non-prismatic channels (Rezaei \& Knight 2009 and Chlebek 2009). Knight et al. (2007) and Tang \& Knight (2008b) have shown that the SKM can satisfactorily be applied to inbank symmetrical channels (including trapezoidal and single section with a vertical wall channels). Furthermore, the SKM methodology was employed in Conveyance Estimation System (CES) software, which provides a practical methodology for predicting velocity and estimating flow capacity in rivers, McGahey et al. (2006).

Although the SKM method was frequently used for estimating the depth-averaged velocity in compound channels with varying width ratios, relative depths, relative roughness and main channel side slopes, there have been relatively few studies conducted to examine the 
applicability of the method on the channels with narrow floodplains. Such a narrow floodplain channels can be existing in many cases and it would be more factual to modelling them as a compound channel than be approximating as a simple trapezoidal cross-section as shown in Figure 1.

\section{[Figure 1 can be inserted here]}

Thus, the current research presents the application of SKM to determine the depth-averaged velocity and boundary shear stress distribution in compound channels with narrow floodplains having different roughness conditions. An analysis of calibrating the key parameters $(f, \lambda$ and $\Gamma)$ used in the model was also performed to establish modified calibration expressions that are fit to narrow floodplain channels. To perform the analysis, two series of experiments were conducted on an asymmetric compound channel with a narrow floodplain.

\section{Depth-averaged modeling using SKM method}

The depth-averaged simulations of flows in the compound channels considered in the present study was carried out by using Shiono and Knight method (SKM). Therefore, it is necessary that a detailed description of the SKM method is given first.

According to this method, Navier-Stokes equation for steady and uniform flow in streamwise direction is combined with the continuity equation to give the following equation (Shiono and Knight 1991):

$$
\rho\left[\frac{\partial}{\partial y}(U V)+\frac{\partial}{\partial z}(U W)\right]=\rho g S_{o}+\frac{\partial \tau_{y x}}{\partial y}+\frac{\partial \tau_{z x}}{\partial z}
$$

where $U, V$ and $W$ are the temporal mean velocity components in $x, y$ and $z$ direction, $x$ is streamwise axis that is parallel to the channel bed; $y$ is lateral and $z$ is normal to the bed, $\rho$ is fluid density, $g$ is gravitational acceleration; $S_{o}$ is channel bed slope, $\tau_{y x}$ and $\tau_{z x}$ are Reynolds stresses on planes perpendicular to the $\mathrm{y}$ and $\mathrm{z}$ directions, respectively. In the depth-averaged modeling, the depth-averaged velocity, bed shear stress and depth-averaged viscosity are given by:

$$
U_{d}=\frac{1}{H} \int_{0}^{H} U d z ; \quad \tau_{b}=\left(\frac{f}{8}\right) \rho U_{d}^{2} ; \quad \varepsilon_{y x}=\lambda U_{*} H
$$


where $U_{d}$ is depth-averaged velocity, $H$ is local water depth, $\tau_{b}$ is local bed shear stress, $U_{*}$ is shear velocity $\left(=\sqrt{\tau_{b} / \rho}\right), f$ is friction factor and $\lambda$ is defined as dimensionless eddy viscosity. The Reynolds shear stress $\tau_{y x}$ acting on lateral planes can be expressed based on the eddy viscosity theory as follows:

$$
\tau_{y x}=\rho \varepsilon_{y x} \frac{\partial U_{d}}{\partial y}
$$

where $\varepsilon_{y x}$ is the depth-averaged eddy viscosity.

Shiono and Knight (1991) simplified Eq. (1) by making use of the expressions in Eqs. (2 \& 3) and then integrated the equation over the total flow depth to obtain the depth-averaged form of Eq. (1):

$$
\rho g H S_{o}-\rho \frac{f}{8} U_{d}^{2}\left(1+\frac{1}{S^{2}}\right)^{\frac{1}{2}}+\frac{\partial}{\partial y}\left[\rho \lambda H^{2}\left(\frac{f}{8}\right)^{1 / 2} U_{d} \frac{\partial U_{d}}{\partial y}\right]=\frac{\partial}{\partial y}\left[H(\rho U V)_{d}\right]
$$

Equation (4) is the basic form of Shiono and Knight method (SKM) that is widely used for depth-averaged calculations in simple and compound channel flows.

The term on the right-hand side of Eq. (4) accounts for the effect of the secondary flow on the primary flow. Shiono and Knight (1991) observed that the lateral gradient of the secondary flow term $\left[H(\rho U V)_{d}\right]$ decreases approximately linearly in the main channel and linearly increases on the floodplains. Therefore, they suggested that a constant value of secondary flow term, which is referred to as secondary flow parameter $(\Gamma)$ can be allocated for main channel and floodplains. Thus, Eq. (4) can be rewritten as follows:

$$
\rho g H S_{o}-\rho \frac{f}{8} U_{d}^{2}\left(1+\frac{1}{S^{2}}\right)^{\frac{1}{2}}+\frac{\partial}{\partial y}\left[\rho \lambda H^{2}\left(\frac{f}{8}\right)^{1 / 2} U_{d} \frac{\partial U_{d}}{\partial y}\right]=\Gamma
$$

For a rectangular compound channel such as a channel considered in this study, Eq. (5) can be solved analytically for $U_{d}$ distribution over a flow region with a constant depth and the solution is given as follows (Shiono and Knight, 1991):

$$
U_{d}=\left[A_{1} e^{\gamma y}+A_{2} e^{-\gamma y}+k\right]^{1 / 2}
$$

where $\gamma$ and $k$ are given as follows: 


$$
k=\frac{8 g S_{o} H}{f}(1-\beta) ; \quad \beta=\frac{\Gamma}{\rho g H S_{o}} ; \quad \gamma=\sqrt{\frac{2}{\lambda}}\left(\frac{f}{8}\right)^{1 / 4} \frac{1}{H}
$$

$A_{1}$ and $A_{2}$ are unknown constants and need to be determined through imposing appropriate boundary conditions on the solution domain.

The accuracy of the SKM results was found to depend substantially on the three depth-averaged calibration coefficients, which includes local bed friction (the Darcy-Weisbach friction factor) $f$; the dimensionless eddy viscosity $\lambda$ and the transverse gradient of secondary flow term $\Gamma$. In the compound channel with wide floodplains, different methods have been suggested to calibrate each one of these three coefficients. The friction factor is often assumed to be constant in each panel and may be back calculated using $\left(f=8 \tau_{b} / U_{d}^{2}\right)$, where the mean depth-averaged velocity $U_{d}$ and shear stress $\tau_{b}$ are measured for each panel (Knight et al. 2007). The dimensionless eddy viscosity for the main channel $\left(\lambda_{m c}\right)$ is usually taken as 0.07 for experimental channels and from 0.24 to 0.5 for natural channels (Knight and Shiono 1990; Shiono and Knight 1991; James and Wark 1992). The value of the dimensionless eddy viscosity for the floodplain $\left(\lambda_{f p}\right)$ is mostly predicted by the expression proposed by Abril and Knight (2004) and given as follows:

$$
\lambda_{f p}=\lambda_{m c}\left(1.2 D_{r}^{1.44}-0.2\right)
$$

where $D_{r}$ is the relative depth and defined as the ratio between the flow depth of the floodplain to that of the main channel. The secondary flow parameter $(\Gamma)$ can be defined as a fraction of the bed shear stresses $\left(\rho g H S_{o}\right)$, (Abril 1997; Abril and Knight 2004 and Omran 2005). The simplification for the secondary flow term may also be made by expressing the term of $V_{d}$ as a function of the $U_{d}$, (Ervine et al. 2000). The assumption is that the product of the local $U_{d}$ and $V_{d}$ velocities produce a profile that is similar to that of the squared depth-averaged streamwise velocity. Therefore, temporal mean velocities $\left[(U V)_{d}\right]$ in the secondary flow term, i.e. the righthand side of Eq. (4), can be rewritten as the fraction of the depth-averaged velocity $\mathrm{Ud}$ in the form:

$$
(U V)_{d}=K U_{d}^{2}
$$

where $K$ is an empirical coefficient that relies on the geometry and boundary roughness. 
However, using the expressions suggested for a compound channel with wide floodplains to calculate the SKM parameters may not give the calibrated values that are proper for a compound channel with narrow floodplains. In this research, the conventional approaches discussed above are checked whether they can also be applicable to the compound channel with narrow floodplains and improved if necessary.

\section{Details of Experiments}

In this study, two different sets of experiments were undertaken in an asymmetric compound channel with the floodplain being narrower than the main channel. The ratio of the floodplain width to the main channel width is 0.50 . The compound channel was constructed inside a flume that has an overall length of $9 \mathrm{~m}$ using smooth plywood sheets. Figure 2 shows the setup of the laboratory flume used in the experiments.

\section{[Figure 2 can be inserted here]}

In both sets of the experiments, the compound channel had a total inside width $(B)$ of $0.3 \mathrm{~m}$ and the main channel width $(b)$ of $0.2 \mathrm{~m}$, as shown in Figure 3. In all test cases, the bed slope of the channels was fixed at about $0.0070 \mathrm{~m} / \mathrm{m}$. The flow was adjusted to achieve uniform flow at a specified normal depth by using an adjustable tailgate at the downstream end of the flume. In a laboratory flume, the ability to establish and recognize uniform flow may be difficult due to the limited length of the flume. However, the best approximation for uniform conditions is when the average water surface level lies at the correct distance above the bed level and both the water and bed levels have the same slope. In the present work, this approximate approach was adopted to establish the uniform condition and determine the normal depth by measuring several profiles of M1 backwater and M2 drawdown curves. The tailgate setting which gave a mean water level slope equal to the flume bed slope was interpolated from two asymptotic M1 and M2 profiles. Experimental conditions for both sets of experiments are listed in Table 1.

\section{[Figure 3 can be inserted here]}

In the first set of experiments that includes the cases CS1, CS2, CS3 and CS4, both the main channel and the floodplain had smooth boundaries and the height of the floodplain $(h)$ was 60 $\mathrm{mm}$. The total flow depth was changed from $80 \mathrm{~mm}$ to $120 \mathrm{~mm}$ to cover a range of relative depth $\operatorname{Dr}[=(H-h) / H]$ from 0.25 to 0.50 . In these smooth floodplain cases, the Manning coefficients $(n)$ 
in the main channel and floodplain were estimated as about 0.009 by applying the Manning equation on normal depth measurements for uniform flows. In most river flow cases, Manning's $n$ has less variation with discharge (Ladson et al. 2013). Therefore, the value of $n$ was assumed to be constant for all discharges considered here.

On the other hand, the cases considered in the second set (i.e. CR1, CR2, CR02 and CR3) had a rough bed on the bottom of the floodplain while the main channel was kept smooth. The height of the floodplain $(h)$ was $75 \mathrm{~mm}$. A single layer of gravel particles with mean size of approximately $6.0 \mathrm{~mm}$ was glued over the bed of the floodplain to create a rough surface. For a bed made up of a single layer of uniform grains (sand or gravel), the equivalent roughness $\left(K_{s}\right)$ is conventionally estimated to be on the order of the size of the grains that make up this layer (López \& Barragán, 2008). Due to the non-uniformity of grain shape and the irregular distribution of gravels when gluing them, the roughness height $K s$ was estimated to be equal to the mean size of gravels $(\approx 6.0 \mathrm{~mm})$ in the present work. For the smooth main channel, $K_{s}$ was calculated based on the Manning's $n$ using the relationship proposed by Ackers (1991) and given by Eq. (22).

\section{[Table 1 can be inserted here]}

For measuring the depth-averaged velocity, a Pitot tube with a diameter of $3.2 \mathrm{~mm}$ and with 4 holes $(\varphi 0.75 \mathrm{~mm}$ ) was used in all test cases. The Pitot tube was connected to the low-range digital pressure transducer (Comark C9551/SIL, 0 to $\pm 140 \mathrm{mbar}$ ), to measure the pressure difference $(\Delta p)$ between the static and dynamic pressures. $\Delta p$ obtained from the output of the transducer was then used to calculate the velocity by applying Bernoulli's concept. Fifteen vertical velocity profiles spaced laterally $20 \mathrm{~mm}$ were measured to obtain the depth-averaged velocity distribution over the entire cross section. In each vertical profile in the floodplain, depthaveraged velocity was established from point velocity measurements at an elevation of 0.4 times the flow depth. In the main channel, eight-point measurements equally spaced along each vertical profile were taken to obtain the depth-averaged velocity. The resulting measured velocity distributions were integrated over the cross section to produce discharges that should be within $\pm 4 \%$ of the discharges measured by the calibrated electromagnetic flow meter. If the difference between the integrated and measured discharges was higher than $4 \%$, the experiments were repeated. 
In the present research, the boundary shear stress was determined by using Preston tube, which is really a pitot tube with outer dimeter of $d=3.2 \mathrm{~mm}$. Boundary shear measurements along the perimeter of main channel and flood plain were taken with around $2 \mathrm{~cm}$ intervals. Two methods of calibration were used in measuring the boundary shear stress. For smooth boundaries, the calibration curve suggested by Patel (1965) was used. Based on Patel calibration method, which has been used for smooth boundaries, the following relationship is given to convert thee pressure readings recorded by Pitot (or Preston) tube to boundary shear stresses:

$$
\begin{gathered}
y_{*}=0.50 x_{*}+0.037, \quad \text { for } 0 \leq x_{*} \leq 2.90 \\
y_{*}=-0.0060 x_{*}^{3}+0.1437 x_{*}^{2}-0.1381 x_{*}+0.8287, \text { for } 2.90 \leq x_{*} \leq 5.60 \\
x_{*}=y_{*}+2 \log _{10}\left(1.95 y_{*}+4.02\right), \quad \text { for } 5.60 \leq x_{*} \leq 7.60
\end{gathered}
$$

where, the two non-dimensional parameters $x_{*}$ and $y_{*}$ are defined as follows:

$$
x_{*}=\log _{10}\left(\frac{\Delta P d^{2}}{4 \rho v^{2}}\right) \text { and } y_{*}=\log _{10}\left(\frac{\tau_{b} d^{2}}{4 \rho v^{2}}\right)
$$

in which, $d$ is the outer diameter of the tube, $\rho$ is the density of the flow, $v$ is the kinematic viscosity of the fluid.

For rough boundaries, the simple calibration equation developed by Wu \& Rajaratnam (2000) was used. For a given roughness and Pitot (or Preston) tube, Wu \& Rajaratnam (2000) proposed a simple calibration expression by which the shear velocity $\left(u_{*}\right)$, which equal to $\left(\sqrt{\tau_{b} / \rho}\right)$, can be calculated:

$$
R_{o}=A R_{s}+a R_{s}+b
$$

where $R_{o}, R_{S}$ and $A$ are given as follows:

$$
A=5.75 \log _{10}\left(z_{o} / K_{s}\right), \quad R_{o}=u_{o} K_{s} / v \text { and } R_{s}=u_{*} K_{s} / v
$$

in which $u_{o}$ is the velocity at the centre of the tube, $K_{s}$ is the equivalent roughness height, $z_{o}$ is the distance from the centre of the tube to the datum of the rough bed. $a$ and $b$ are constants which their values are found to be dependent on the values of $R_{S}$. For $R_{S}>70$, as in the test cases considered in the present study, the coefficients $a$ and $b$ have the values of 8.5 and $0.0,(\mathrm{Wu} \&$ Rajaratnam, 2000). 
The total shear force per unit length normal to flow cross section was calculated by integrating the shear stress measurements over the entire perimeter. The integrated total shear then compared with the theoretical shear force based on the energy gradient approach $\left(\rho g A S_{o}\right)$. The error values obtained from two methods were found within $\pm 2.5 \%$ for smooth boundaries and $\pm 5 \%$ for rough boundaries.

\section{Applying SKM model to the case study}

\subsection{Number of panels and Boundary conditions}

To apply the analytical depth-averaged model based on SKM method, the channel is first divided into a sufficient number of panels and then the appropriate boundary conditions are imposed on the panels. For all test cases considered here, the asymmetric rectangular compound channels were divided into only two panels, one for the main channel and the other for the floodplain region. Because all channels simulated in this work have a regular and uncomplicated cross section, the simulations were restricted to a small number of panels. Furthermore, choosing the minimum number of panels with a reasonable useful output are typically acceptable for practical purposes since the SKM method itself is only an approximate one. However, for modeling some particular flow cases where greater precision is required, more number of panels (4 or more) may be necessary, (Knight et al. 2009).

To determine the integration constants $(A 1, A 2)$ in Eq. (6), the boundary conditions need to be applied between the panels and at the channel edges. In general, there are different types of boundary conditions can be applied in the applications of SKM model. These may include:

(1) the no-slip condition at the remote edges of the channel (Figure 3),

$$
\left(U_{d}\right)_{y=0}^{i}=\left(U_{d}\right)_{y=B}^{i+1}=0
$$

(2) the continuities of the velocity $\left(U_{d}\right)$ and its gradient gradients $\left(\partial U_{d} / \partial y\right)$ at the interface between the adjacent panels (Knight et al. 2004; and Liao \& Knight 2007),

$$
\left(U_{d}\right)_{y=b}^{i}=\left(U_{d}\right)_{y=b}^{i+1} ; \quad\left(\frac{\partial U_{d}}{\partial y}\right)_{y=b}^{i}=\left(\frac{\partial U_{d}}{\partial y}\right)_{y=b}^{i+1}
$$

(3) the continuities of $H U_{d}$ and the lateral gradient of $\partial H U_{d} / \partial y$ at the interface between the adjacent panels (Shiono and Knight 1991), 


$$
\left(H U_{d}\right)_{y=b}^{i}=\left(H U_{d}\right)_{y=b}^{i+1} ; \quad\left(\frac{\partial H U_{d}}{\partial y}\right)_{y=b}^{i}=\left(\frac{\partial H U_{d}}{\partial y}\right)_{y=b}^{i+1}
$$

However, Tang and Knight (2008b) pointed out that there are considerable difficulties in specifying boundary conditions for rectangular compound channels where the internal wall between the floodplain and the main channel is vertical. they analyzed a wide selection of data and suggested that the continuity of unit force was technically the most suitable for the cases of vertical internal walls. Based on their analysis, the following relationship for the continuity of unit force was proposed to apply at the vertical wall between the main channel and the floodplain:

$$
\left(H \bar{\tau}_{y x}\right)_{y=b}^{i}+h \tau_{w}=\left(H \bar{\tau}_{y x}\right)_{y=b}^{i+1}
$$

where $\tau_{w}$ is averaged shear stress at the internal wall. By employing the expression that relate explicitly the Reynolds stresses with mean velocity gradients, Eq. (17) can be rewritten as follows:

$$
\begin{gathered}
\left(\phi \frac{\partial U_{d}^{2}}{\partial y}\right)_{y=b}^{i}=\left(\phi \frac{\partial U_{d}^{2}}{\partial y}\right)_{y=b}^{i+1}-h \tau_{w} \\
\phi=\frac{1}{2} \rho \lambda H^{2} \sqrt{f / 8} \text { and } \tau_{w}=\rho f_{w}\left(U_{d}^{2}\right)_{y=b} / 8
\end{gathered}
$$

The boundary condition given by Eq. (18) has a drawback because the wall shear stress $\left(\tau_{w}\right)$ at the vertical wall needs to be known in prior. Therefore, the wall shear stress $\left(\tau_{w}\right)$ is approximated by using the friction factor in the main channel and the depth-averaged velocity at the interface as given by Eq. (19).

Tang and Knight (2008b), based on their investigation into selecting suitable boundary conditions for a channel with an internal vertical wall, confirmed that the boundary condition given in Eq. (18) is the most appropriate one. Therefore, this type of boundary condition was applied at the vertical wall between the main channel and floodplain, while the no-slip condition was imposed at the remote edges. 


\subsection{Calibrating the model parameters}

In addition to choosing the number of panels, the appropriate choice of values for the calibration parameters $(f, \lambda, \Gamma)$ for each panel are also essential for correct calculations. Based on the principle involved in the SKM method, the friction parameter $f$ is usually determined for each panel through the use of the Colebrook-White equation (Shiono and Knight 1991):

$$
\frac{1}{\sqrt{f}}=-2.03 \log _{10}\left[\frac{K_{s}}{12.27 R}+\frac{3.09}{R e \sqrt{f}}\right]
$$

Where $K_{s}$ is the roughness height, $R e$ is the Reynolds Number and $R$ is the hydraulic radius. This equation considers the full three-dimensional flow effects throughout the depth of flow. In spite of that, the Colebrook-White equation evaluates a lateral distribution of $f$ at each flow region based on an iterative estimation. Therefore, a simpler formulation of the Colebrook-White equation which is developed by Rameshwaran and Shiono (2007) was used here for estimating the value of $f$ for both the main channel and the floodplain panels. This equation can be written as follows:

$$
f=\left[-2.0 \log _{10}\left(\frac{3.02 v}{\sqrt{128 g H^{3} S_{o}}}+\frac{K_{s}}{12.3 H}\right)\right]^{-2}
$$

where $v$ is kinematic viscosity. If the values of $K_{s}$ are not available, they can be calculated from the relationship that relates the Manning coefficients $n$ to the surface roughness (Ackers 1991):

$$
K_{s}=(8.25 \sqrt{g} n)^{6}
$$

The resistance relationship given by Eq. (21) was used by several researchers, e.g. Sun X. (2007) and Yang et al. (2012), for estimating the friction factor in rectangular compound channels and a reasonably good agreement has been noticed between the calculated and measured values for $f$. The most common method of calibrating the dimensionless eddy viscosity coefficient $(\lambda)$ is the method of expressing the ratio of this coefficient between the floodplain and the main channels $\left(\lambda_{f p} / \lambda_{m c}\right)$ in terms of the relative depth $\mathrm{Dr}$. In this method, the coefficient of the dimensionless eddy viscosity $\left(\lambda_{f p}\right)$ in the floodplain is determined by estimating a value for the coefficient $\left(\lambda_{m c}\right)$ in the main channel which is usually independent of the flow depth. The value of $\lambda_{m c}=0.07$ is 
often used as the initial value in the main channel, with the following general expression used for the ratio $\left(\lambda_{f p} / \lambda_{m c}\right)$ :

$$
\frac{\lambda_{f p}}{\lambda_{m c}}=a D r^{b}-c
$$

Equation (23) was proposed by Abril \& Knight (2004) based experiments conducted by Knight $\&$ Abril experiments (1996) on compound channels with a wide floodplain. They suggested that the coefficients $a, b$ and $c$ can take values as $1.20,-1.44$ and 0.20 respectively. It should be pointed out that the initial dimensionless eddy viscosity value for the main channel, $\lambda_{m c}$, was taken to be same as the depth-averaged value assumed for a wide channel $(k / 6=0.41 / 6 \approx 0.07$, where $k=$ von Karman's constant). Although the values of $\lambda_{f p}$ provided by Eq. (23) were consistent with the experimental data of Knight \& Abril (1996), it is not strictly applicable to experiments considered here. It is thought that the transverse variation of the dimensionless eddy viscosity $(\lambda)$ may be dependent on the roughness and width of the floodplains (Fernandes et al. 2014). Therefore, further investigation may be required to check whether Eq. (23) with the coefficients values recommended by Abril \& Knight (2004) can successfully be used in the simulations of various compound channels that have different floodplains in terms of width and bottom roughness, as shown elsewhere.

The calibration of the secondary flow parameter $(\Gamma)$ needs to detailed measurements of threedimensional velocities at the junction zone between the main channel and the floodplain (Shan et al. 2016). Nevertheless, as mentioned previously, the gradient of the secondary flow term is constant within a certain zone, allowing constant values of $\Gamma$ to be assigned to each panel. Hence, for a given channel, the constant values of $\Gamma$ can be set for the main channel and the floodplain regions. For the simulations in the present study, the conventional expression which relates the secondary flow term to the average shear stress was used to calibrate $\Gamma$. This expression can be given for the main channel and floodplain as follows:

$$
\begin{gathered}
\Gamma_{m c}=k_{m c}\left(\rho g S_{o} H\right) \\
\Gamma_{f p}=k_{f p}\left(\rho g S_{o} H\right)
\end{gathered}
$$

where $k$ defined as the secondary flow coefficient which is different in the main channel and the floodplain regions. Based on data obtained by Shiono \& Knight (1991) from the Flood Channel 
Facility experiments for different relative depths in a straight compound channel, Abril \& Knight (2004) recommended that $k_{m c}=0.15$ and $k_{f p}=-0.25$ can be used as calibrated values for the secondary flow coefficients. As the secondary current term $\Gamma$ varies with flow depth and geometry of the cross section (Yang et al. 2012) and may depend on the bottom roughness (Fernandes et al. 2014), so it is expected that the standard values of $k$ suggested by Abril \& Knight (2004) may not be fit to the flow cases under consideration. Therefore, this issue has been further discussed in a later section of this article.

\section{Analysis of results}

\subsection{Flow Cases with Smooth Narrow Floodplains}

The analytical SKM model was first applied to the cases of the smooth narrow floodplains (i.e. cases CS1 CS4) using the conventional calibration values of friction factor $(f)$, dimensionless eddy viscosity $(\lambda)$ and secondary flow parameter $(\Gamma)$. The predicted results for the depthaveraged velocity $\left(U_{d}\right)$ and the bed shear stress $\left(\tau_{b}\right)$ are plotted in Figures $4 \& 5$ and referred to as SKM1 (dashed lines). For the SKM1 application, $f$ was estimated from the modified DarcyWeisbach equation, Eq. (21). The dimensionless eddy viscosity parameter for the main channel $\left(\lambda_{m c}\right)$ was assumed to be $(0.07)$ and that for the floodplain $\left(\lambda_{f p}\right)$ was computed from Eq. (23) using its conventional form, i.e. $\lambda_{f p}=\lambda_{m c}\left(1.20 D r^{-1.44}-0.2\right)$. The secondary flow parameters for the main channel and floodplain were calculated as a fraction of the bed shear stress from (Eqs. $24 \& 25$ ) with values of $k_{m c}=0.15$ and $k_{f p}=-0.25$ as recommended by Abril and Knight (2004).

\section{[Figure 4 can be inserted here]}

[Figure 5 can be inserted here]

From Figure 4, it can be seen that for all flow cases, the analytical solutions for $U_{d}$ in the main channel agree reasonably well with the experimental data. However, it seems that the simulated distributions for $U_{d}$ in the floodplain region do not match closely with the measured ones, particularly for flow cases of relatively high relative depth $(D r>0.33)$. In that region, the lateral distributions of $U_{d}$ are underpredicted as clearly shown for the cases CS3 and CS4 (Figure 4c and $4 d)$. 
Similar trends for boundary shear stress $\left(\tau_{b}\right)$ from SKM1 application can be observed in Figure 5. There is a good agreement between the simulated results (dashed lines) and experimental data for $\tau_{b}$ in the main channel, but the results show that the simulated distributions of $\tau_{b}$ are underpredicted in the floodplain, in particular for high flow cases $(D r>0.33)$. It should be noted that the agreement of the predicted results for $U_{d}$ with the experimental data is relatively better than that for the $\tau_{b}$ results. This is because $\tau_{b}$ is related to $U_{d}$ by a quadratic relationship, consequently the error in prediction of $\tau_{b}$ is larger.

The results obtained from SKM1 application indicates that the conventional values of $k_{f p}=-0.25$ may not be appropriate for simulating high flows over a smooth narrow floodplain. Because the volume and mass of water in the narrow floodplain is relatively smaller than that in a wide floodplain, it would be expected that the effects of the secondary circulations between the main channel and the floodplain on the primary velocity in the floodplain are higher than that in wide floodplain channels. Thus, the value of $k_{f p}$ should be increased to account for the increased effects of secondary currents in the floodplain region.

The smooth floodplain cases (CS1 \& CS4) were re-simulated, where the friction factor and the $\lambda$ values were kept unchanged and the $k_{f p}$ was only adjusted to provide the best fit to the measured depth-averaged velocity $\left(U_{d}\right)$ and boundary shear $\left(\tau_{b}\right)$ distributions. The results for $U_{d}$ and $\tau_{b}$ from these simulations are referred to as SKM2 and plotted as solid lines in Figures $4 \& 5$. It was found that only increasing the value of $k_{f p}$ was sufficient to improve the simulated results for both $U_{d}$ and $\tau_{b}$ over the floodplains. The analytical results for the floodplain improved appreciably when secondary flow coefficient $\left(k_{f p}\right)$ was doubled from -0.25 to -0.50 in the conventional expression proposed by Abril and Knight (2004).

In the light of the analysis above, it can be concluded that similar to the wide floodplains, the secondary flows effects on the streamwise velocity and boundary shear stress can be estimated as a fraction of boundary shear stress in the cases of smooth narrow floodplain such as channels considered here. However, for the narrow smooth floodplains the secondary flow effects are more significant than that in the case of wide floodplains and the shear generated due to the secondary flow may be about $50 \%$ from the mean bed shear stress. 


\subsection{Flow Cases with Rough Narrow Floodplains}

Figures 6 and 7 show the simulated results for $U_{d}$ and $\tau_{b}$ for all rough cases (CR1 CR4), together with experimental data. In applying the model to the rough floodplain cases, the eddy viscosity coefficients and the secondary flow coefficients in the main channel and floodplain were first assumed to be as same as their conventional values that may be applied for shallow and wide floodplain channels, i.e. $\lambda_{m c}=0.07, k_{m c}=0.15$ and $k_{f p}=-0.25$. The friction factor was estimated from the modified Darcy-Weisbach equation. The simulated results obtained using the conventional values of model parameters are plotted as dashed lines and referred to as SKM1.

For SKM1 simulation, it appears that the computed velocity $\left(U_{d}\right)$ in the main channel is greater than the experimental data and its gradient is steeper than the one from the experiments, particularly for low flow cases (Figure 6a and 6b). For the higher flow cases $(D r>0.33)$, the differences between the simulated and measured profiles are relatively small. Figure 7 also shows similar trend for the simulated results for $\tau_{b}$, where $\tau_{b}$ profiles from SKM1 predictions are fairly in good agreement with the measured data in the floodplain regions but are not over the main channels. The simulated distribution of $\tau_{b}$ is overpredicted in the main channel due to the overprediction of the simulated velocity $\left(U_{d}\right)$. This suggest that the assumed values of the eddy viscosity for the main channel may be underestimated, leading to the simulated profiles of $U_{d}$ and $\tau_{b}$ that are overpredicted and steeper in that region.

\section{[Figure 6 can be inserted here]}

\section{[Figure 7 can be inserted here]}

Figures 6 and 7 shows that the predicted results for both $U_{d}$ and $\tau_{b}$ can be improved when only the eddy viscosity parameter for the main channel $\left(\lambda_{m c}\right)$ is increased to about (0.09), as shown in SKM3 profiles (solid line). As a result of $\lambda$ adjustment, the SKM3 distribution in comparison with SKM1 agrees better with the experimental data; in particular, at the main channel where the profile gradient matches better to that of the measured profile.

It should be noted that the results were improved without needing to modify the conventional values for secondary flow coefficients $k_{m c}$ and $k_{f p}$. This indicates that the conventional calibrated values for secondary flow parameters can effectively be used for simulating rough narrow floodplain channels. As mentioned previously, the depth-averaged velocities term $\left[(U V)_{d}\right]$ can 
be related to the depth-averaged streamwise velocity $U_{d}$ (Ervine et al. 2000), so the secondary flow effect is expected to decrease when the $U_{d}$ decrease because of increased roughness. This means that in the case of rough narrow floodplains, the secondary flow has a smaller contribution in energy loses compared to the lateral shear. Thus, it is suggested that the secondary flow coefficients can be estimated as their conventional values which are recommended for wide floodplain channels.

\section{Conclusions}

In this study, 2D depth-averaged simulations based on Shiono and Knight method (SKM) were performed to simulate flows in asymmetric compound channels with narrow floodplains. The applicability of Shiono and Knight method (SKM) to predict the primary velocity $\left(U_{d}\right)$ and boundary shear stress $\left(\tau_{b}\right)$ was explored. For SKM modelling, a number of empirical input parameters are required, including the bed friction factor $f$, the dimensionless eddy viscosity coefficient $\lambda$ and the secondary flow parameter $\Gamma$. The latter two require calibration or reliable estimates to produce accurate predictions.

Two different sets of experiments were conducted, one on rectangular compound channels with smooth floodplains and the other on channels with rough floodplains. Flow cases considered in the experiments were then simulated by using the SKM model. For compound channels with smooth narrow floodplains, the calibration methodology developed by Abril and Knight (2004), which is referred as a conventional method, was found to produce underpredicted values of $U_{d}$ and $\tau_{b}$ in the floodplain regions, particularly for the cases of relative depths $(D r)$ larger than 0.33 . However, the simulated results can be improved after the secondary flow coefficients in the floodplain $\left(k_{f p}\right)$ are increased from -0.25 to -0.50 . This demonstrates that the secondary flow effect is more significant in smooth narrow floodplains compared to wide floodplains, and the secondary flow force would be estimated to be about 0.5 of the mean bed shear force.

For compound channels with rough narrow floodplains, the conventional values of the secondary flow coefficients can be used for predicting the primary velocity and boundary shear stress in both flow regions, but the values of eddy viscosity coefficient $\left(\lambda_{m c}\right)$ needs to be adjusted in order to improve the simulation results in the main channel. In general, the conventional expressions proposed to describe the effects of secondary currents and lateral shear in wide 
floodplain channels cannot be used as a universal method to obtain predictors of SKM coefficients in narrow floodplain channels. 


\section{References}

Abril, B. (1997). Numerical modelling of turbulent flow, sediment transport and flood routing using the finite element method (Doctoral dissertation, University of Birmingham).

Abril, J. B., \& Knight, D. W. (2004). Stage-discharge prediction for rivers in flood applying a depth-averaged model. Journal of Hydraulic Research, 42(6), 616-629.

Ackers, P. (1991). Hydraulic design of straight compound channels. Rep. SR 281, Hydraulics Research Ltd., Wallingford, U.K.

Chlebek, J. (2009). Modelling of simple prismatic channels with varying roughness using the SKM and a study of flows in smooth non-prismatic channels with skewed floodplains (Doctoral dissertation, University of Birmingham).

Devi, K., \& Khatua, K. K. (2016). Prediction of depth averaged velocity and boundary shear distribution of a compound channel based on the mixing layer theory. Flow Measurement and Instrumentation, 50, 147-157.

Ervine, D. A., \& Ellis, J. (1987). Experimental and computational aspects of overbank floodplain flow. Earth and Environmental Science Transactions of The Royal Society of Edinburgh, 78(4), 315-325.

Ervine, D. A., Babaeyan-Koopaei, K., \& Sellin, R. H. (2000). Two-dimensional solution for straight and meandering overbank flows. Journal of Hydraulic Engineering, 126(9), 653-669.

Fernandes, J. N., Leal, J. B., \& Cardoso, A. H. (2014). Improvement of the lateral distribution method based on the mixing layer theory. Advances in water resources, 69, 159-167.

James, C. S., \& Wark, J. B. (1992). Conveyance estimation for meandering channels. Project Rep., HR Wallingford, Wallingford, Oxfordshire, U.K., 255.

Knight, D. W., \& Boris Abril C, J. (1996). Refined calibration of a depth-averaged model for turbulent flow in a compound channel. Proceedings of the Institution of Civil Engineers-Water Maritime and Energy, 118(3), 151-159.

Knight, D. W., \& Tang, X. (2008). Zonal discharges and boundary shear in prismatic channels. Proceedings of the Institution of Civil Engineers-Engineering and Computational Mechanics, 161(2), 59-68. 
Knight, D. W., McGahey, C., Lamb, R., \& Samuels, P. (2009). Practical channel hydraulics: Roughness, conveyance and afflux. CRC Press. Florida, United States.

Knight, D. W., Omran, M., \& Abril, J. B. (2004). Boundary conditions between panels in depthaveraged flow models revisited. In River Flow 2004, Proc., 2nd Int. Conf. on Fluvial Hydraulics, 23-25 June, Napoli, Italy (Vol. 1, pp. 371-380).

Knight, D. W., Omran, M., \& Tang, X. (2007). Modeling depth-averaged velocity and boundary shear in trapezoidal channels with secondary flows. Journal of Hydraulic Engineering, 133(1), 39-47.

Ladson, A. R., Lang, S. M., Smart, G. M., Anderson, B. G., \& Rutherfurd, I. D. (2013). Flow resistance in four rivers in Victoria, Australia. Australasian Journal of Water Resources, 16(2), 173-180.

Lambert, M. F., \& Sellin, R. H. J. (1996). Discharge prediction in straight compound channels using the mixing length concept. Journal of Hydraulic Research, 34(3), 381-394.

Liao, H., \& Knight, D. W. (2007). Analytic stage-discharge formulas for flow in straight prismatic channels. Journal of Hydraulic Engineering, 133(10), 1111-1122.

Liu, C., Wright, N., Liu, X., \& Yang, K. (2014). An analytical model for lateral depth-averaged velocity distributions along a meander in curved compound channels. Advances in water resources, 74, 26-43.

López, R., \& Barragán, J. (2008). Equivalent roughness of gravel-bed rivers. Journal of Hydraulic Engineering, 134(6), 847-851.

McGahey, C., Samuels, P. G., \& Knight, D. W. (2006). A practical approach to estimating the flow capacity of rivers-application and analysis (pp. 303-312). London: Taylor and Francis.

Omran, M. (2005). Modelling stage-discharge curves, velocity and boundary shear stress distributions in natural and artificial channels using a depth-averaged approach. PhD Thesis. The University of Birmingham, UK.

Rameshwaran, P., \& Shiono, K. (2007). Quasi two-dimensional model for straight overbank flows through emergent. Journal of Hydraulic Research, 45(3), 302-315. 
Rezaei, B., \& Knight, D. W. (2009). Application of the Shiono and Knight Method in compound channels with non-prismatic floodplains. Journal of Hydraulic Research, 47(6), 716-726.

Seckin, G., Mamak, M., Atabay, S., \& Omran, M. (2009). Discharge estimation in compound channels with fixed and mobile bed. Sadhana, 34(6), 923-945.

Shan, Yu-qi, L. I. U. Chao, Mao-kang Luo, and Ke-jun Yang. "A simple method for estimating bed shear stress in smooth and vegetated compound channels." Journal of Hydrodynamics, Ser. B 28, no. 3 (2016): 497-505.

Shiono, K., \& Knight, D. W. (1988). Two-dimensional analytical solution for a compound channel. In Proc., 3rd Int. Symp. on refined flow modeling and turbulence measurements (pp. 503-510).

Shiono, K., \& Knight, D. W. (1991). Turbulent open-channel flows with variable depth across the channel. Journal of Fluid Mechanics, 222, 617-646.

Spooner, J., \& Shiono, K. (2003). Modelling of meandering channels for overbank flow. In Proceedings of the Institution of Civil Engineers-Water and Maritime Engineering (Vol. 156, No. 3, pp. 225-233). Thomas Telford Ltd.

Sun, X. (2007). Flow characteristics in compound channels with and without vegetation. Doctoral dissertation, Loughborough University.

Tang, X., \& Knight, D. W. (2008a). Lateral depth-averaged velocity distributions and bed shear in rectangular compound channels. Journal of Hydraulic Engineering, 134(9), 1337-1342.

Tang, X., \& Knight, D. W. (2008b). A general model of lateral depth-averaged velocity distributions for open channel flows. Advances in Water Resources, 31(5), 846-857.

Van Prooijen, B. C., Battjes, J. A., \& Uijttewaal, W. S. (2005). Momentum exchange in straight uniform compound channel flow. Journal of hydraulic engineering, 131(3), 175-183.

Wark, J. B., Samuels, P. G., \& Ervine, D. A. (1990). A practical method of estimating velocity and discharge in compound channels. River flood hydraulics, 163-172.

Wu, S., \& Rajaratnam, N. (2000). A simple method for measuring shear stress on rough boundaries. Journal of Hydraulic Research, 38(5), 399-401. 
Yang, K., Nie, R., Liu, X., \& Cao, S. (2012). Modeling depth-averaged velocity and boundary shear stress in rectangular compound channels with secondary flows. Journal of Hydraulic Engineering, 139(1), 76-83. 